\title{
MicroRNA-134 as a potential plasma biomarker for the diagnosis of acute pulmonary embolism
}

\author{
Junjie Xiao ${ }^{1,2 \dagger}$, Zhi-Cheng Jing ${ }^{3 \dagger}$, Patrick T Ellinor ${ }^{4}$, Dandan Liang ${ }^{1,2}$, Hong Zhang ${ }^{1,2}$, Ying Liu ${ }^{1,2}$, Xiaoli Chen , \\ Lei Pan ${ }^{1}$, Robert Lyon ${ }^{5}$, Yi Liư ${ }^{1,2}$, Lu-Ying Peng ${ }^{1,2}$, Xingqun Liang ${ }^{1}$, Yunfu Sun ${ }^{1}$, Laurentiu M Popescu ${ }^{6}$, \\ Gianluigi Condorelli ${ }^{5}$ and Yi-Han Chen ${ }^{1,2^{*}}$
}

\begin{abstract}
Background: Acute pulmonary embolism (APE) remains a diagnostic challenge due to a variable clinical presentation and the lack of a reliable screening tool. MicroRNAs (miRNAs) regulate gene expression in a wide range of pathophysiologic processes. Circulating miRNAs are emerging biomarkers in heart failure, type 2 diabetes and other disease states; however, using plasma miRNAs as biomarkers for the diagnosis of APE is still unknown.

Methods: Thirty-two APE patients, 32 healthy controls, and 22 non-APE patients (reported dyspnea, chest pain, or cough) were enrolled in this study. The TaqMan miRNA microarray was used to identify dysregulated miRNAs in the plasma of APE patients. The TaqMan-based miRNA quantitative real-time reverse transcription polymerase chain reactions were used to validate the dysregulated miRNAs. The receiver-operator characteristic (ROC) curve analysis was conducted to evaluate the diagnostic accuracy of the miRNA identified as the candidate biomarker.
\end{abstract}

Results: Plasma miRNA-134 (miR-134) level was significantly higher in the APE patients than in the healthy controls or non-APE patients. The ROC curve showed that plasma miR-134 was a specific diagnostic predictor of APE with an area under the curve of 0.833 (95\% confidence interval, 0.737 to $0.929 ; \mathrm{P}<0.001$ ).

Conclusions: Our findings indicated that plasma miR-134 could be an important biomarker for the diagnosis of APE. Because of this finding, large-scale investigations are urgently needed to pave the way from basic research to clinical utilization.

\section{Background}

Acute pulmonary embolism (APE) is a common cardiovascular emergency associated with a substantial morbidity and mortality [1-3]. APE accounts for 50, 000 to 100, 000 deaths a year in the United States alone and approximately $10 \%$ of deaths in European hospitals [2,4]. The annual incidence of APE in the United States and Europe is approximately 100 cases per 100, 000 individuals [2,4]. Despite the morbidity associated with APE, the diagnosis is frequently missed due to the variable clinical presentation $[5,6]$.

Diagnostic testing for APE has been extensively studied and ranges from biomarkers, such as the D-dimer assay, to radiologic imaging, including CT angiography, venous ultrasonography, and ultimately the gold standard of

\footnotetext{
* Correspondence: yihanchen@tongji.edu.cn

† Contributed equally

'Key Laboratory of Arrhythmias, Ministry of Education, China (East Hospital,

Tongji University School of Medicine), Shanghai, China

Full list of author information is available at the end of the article
}

pulmonary venous angiography. Although widely used as a screening tool, D-dimer assays are sensitive but not specific for detecting APE [7]. Novel biomarkers with enhanced diagnostic accuracy would greatly facilitate the diagnosis of APE [3].

In recent years, microRNAs (miRNAs) have been found to play crucial roles in many cellular processes, such as development, proliferation, differentiation and apoptosis. MiRNAs are small, endogenous, single-stranded, noncoding RNAs that regulate gene expression by hybridizing to messenger RNAs (mRNAs) and inhibiting mRNA translation or promoting mRNA degradation [8-11]. The expression pattern of many miRNAs is reflective of various pathophysiologic processes and underlies a large number of diseases [8,12-14]. Circulating miRNAs are also emerging as biomarkers in various diseases, including cancer, druginduced liver injury, heart failure, type 2 diabetes, stable angina pectoris, and acute coronary artery syndromes 
[8,12-23]. We therefore sought to explore plasma miRNAs as biomarkers for the diagnosis of APE.

\section{Methods \\ Patient populations}

Between February 2010 and July 2010, patients with a high clinical probability of APE or those with a low/intermediate probability and a positive $\mathrm{D}$-dimer enzymelinked immunosorbent assay test $(>500 \mu \mathrm{g} / \mathrm{L})$ in the Shanghai Pulmonary Hospital, Tongji University underwent testing to confirm APE. In accordance with existing guidelines, these patients underwent a pulmonary angiography, contrast-enhanced computed tomography (CT) pulmonary angiography or a ventilation-perfusion lung scan, alone or in combination [24]. After the diagnosis of APE, transthoracic echocardiography was performed to detect (or exclude) right ventricular dysfunction (i.e., dilation of the right ventricle with an end-diastolic diameter $>30 \mathrm{~mm}$ from the parasternal view, or the right ventricle appearing larger than the left ventricle from the subcostal or apical view) combined with right atrial hypertension (absence of inspiratory collapse of the inferior vena cava) in the absence of left ventricular or mitral valve disease. Serum cardiac troponin I and BNP were used as markers of myocardial injury or right ventricular dysfunction, respectively. Afterwards, risk stratification was conducted according to the guidelines [24]. Briefly, high-risk APE is diagnosed if shock or hypotension occurs. Intermediate-risk APE is confirmed when at least one right ventricular dysfunction or one myocardial injury marker is positive. Low-risk APE is confirmed when all right ventricular dysfunction and myocardial injury markers are negative [24]. During this period, 35 patients were confirmed to have an APE, and the 32 patients that gave informed consent were enrolled in this study. The controls were 32 healthy volunteers. Twentytwo patients ( 7 with pneumonia, 7 with unstable angina pectoris, 3 with acute myocardial infarctions, 2 with lung cancer, 1 with pleurisy, 1 with bronchiectasis and 1 with asthma) who had reported dyspnea, chest pain, or cough were consecutively recruited as the non-APE cases. Approval was obtained from the ethical committees of the Tongji University School of Medicine. All participants gave written informed consent before enrollment in the study.

\section{Plasma sampling and RNA isolation}

At presentation, blood samples for miRNA detection were collected in EDTA-K2 tubes and processed within 1 hour of collection. After a two-step centrifugation $\left(4^{\circ} \mathrm{C}\right.$ at $820 \times g$ for $10 \mathrm{~min}$, then $4^{\circ} \mathrm{C}$ at $16000 \times g$ for $10 \mathrm{~min}$ ), the supernatant was transferred to RNase/DNase-free tubes and stored at $-80^{\circ} \mathrm{C}$.
The total RNA was isolated from the plasma using a mirVana PARIS isolation kit (Ambion, Austin, Texas) according to the manufacturer's instructions for plasma samples. Briefly, $400 \mu \mathrm{L}$ of human plasma was used to extract the total RNA. Each sample was eluted in $100 \mu \mathrm{L}$ of RNAse-free water. Spectrophotometric RNA quantification of human plasma samples was not reliable because of undetermined contaminants with an absorbance peak at $270 \mathrm{~nm}$. However, the quantitative real-time reverse transcription polymerase chain reaction (qRT-PCR) was not affected [12]. Thus, all RNA samples were analyzed for miR-16 expression, a stable endogenous reference miRNA, to assess an approximate yield of RNA extraction and to ensure that comparable amounts of starting material were used in each reverse transcription reaction [25-29].

\section{miRNA expression profile and validation}

miRNAs were reverse-transcribed using the Megaplex Primer Pools (Human Pools A v2.1 and B v2.0), and the products were pre-amplified according to the Applied Biosystems' pre-amplification protocol. For the initial screening, Human TaqMan miRNA microarrays (CardA v2.1 and CardB v2.0, Applied Biosystems) covering 667 small, noncoding RNAs were performed on RNA from the plasma of 10 randomly selected APE patients and 10 randomly selected healthy controls. All steps were performed using a 7900HT Fast Real-Time PCR System (Applied Biosystems, Foster City, CA); the results were expressed as Cts and normalized to the calculated median $\mathrm{Ct}$ of each sample $(\Delta \mathrm{Ct})$. The relative expression was calculated using the comparative $\mathrm{Ct}$ method $\left(2^{-\Delta \Delta \mathrm{Ct}}\right)$. The minimal microarray experiment compliant data were exported to the Gene Expression Omnibus (Platform ID GSE24149).

To minimize the number of false positives, only miRNAs that differed from the healthy controls by more than 10 -fold were considered for subsequent validation by qRT-PCR. Single miRNA expression was determined using TaqMan-based miRNA qRT-PCR (Applied Biosystems, Foster City, CA) according to the manufacturer's instructions. Briefly, the $15-\mu \mathrm{L}$ RT reaction master mix was created with $5 \mu \mathrm{L}$ of the total RNA sample isolated as described above. qRT-PCR was carried out using the 7900 HT Fast Real-Time PCR System on $20 \mu \mathrm{L}$ of PCR master mix containing $10 \mu \mathrm{L}$ of TaqMan $2 \times$ Universal PCR Master Mix, $1 \mu \mathrm{L}$ of TaqMan assay, $4 \mu \mathrm{L}$ of RT products and $5 \mu \mathrm{L}$ of $\mathrm{ddH}_{2} \mathrm{O}$. The qRT-PCR reactions were performed in triplicate, and the signal was collected at the end of every cycle. Due to a lack of generally accepted standards, all qPCR data on single miRNA expression were analyzed as unadjusted $\mathrm{Ct}$ values and standardized to miR-16, which fulfilled the following criteria: detectable in all samples, low dispersion of expression levels and a null 
association with APE. To validate miR-16 as a stable internal reference, its stability during extraction was compared to that of synthetic cel-miR-39, an miRNA of C. elegans that is not present in humans. Twenty-five fmol of synthetic cel-miR-39 were spiked in after adding the Denaturing Solution (provided in the mirVana PARIS isolation kit) to the human plasma samples to avoid degradation by endogenous RNases, and the RNA was extracted. We measured the expression of cel-miR-39, miR-16 and miR134 in 10 APE patients and 10 healthy controls selected at random. Afterwards, the expression of miR-134 in APE patients and healthy controls was compared with miR-16 and cel-miR-39 normalizers, respectively.

\section{Statistical analysis}

Data characterized by a normal distribution were expressed as the mean and standard deviation. Relative gene expression is widely presented using the $2^{-\Delta \Delta C t}$ method [30]. Relative gene expression involves the gene of interest data $\left(\mathrm{Ct}_{\text {gene of interest }}\right)$ relative to an internal control gene $\left(\mathrm{Ct}_{\text {internal control gene }}\right)$, named delta $\mathrm{Ct}$. The calculated delta $\mathrm{Ct} \pm \mathrm{SD}$ for the different groups (APE and non-APE) were compared with the delta $\mathrm{Ct} \pm \mathrm{SD}$ (SD stands for the standard deviation of the average delta $\mathrm{Ct}$ of the group) for the healthy control group and tested for statistical significance. An independent samples $t$-test, Chi-squared test or one-way analysis of variance (oneway ANOVA) was conducted when appropriate. If a significant difference was found, a Bonferroni post-hoc test or Tamhane's T2 post-hoc analysis was conducted to determine which groups differed significantly according to the equal variance criterion. The receiver-operator characteristic (ROC) curve analysis was performed with plasma miRNA distinguishing between APE patients and healthy controls or non-APE patients. The area under the ROC curve (AUC) was estimated to assess the diagnostic accuracy of the miRNA identified. All analyses were performed using SPSS 13.0, and all statistical tests were two-sided. For all analyses, p-values $<0.05$ were considered statistically significant.

\section{Results}

Expression profiles of miRNAs in the plasma of APE patients

Thirty differentially expressed plasma miRNAs were identified in APE patients from healthy controls (Table 1). From these, microRNA-134 (miR-134) and microRNA410 (miR-410) were selected for further validation based on the changes (>10-fold) and probability values $(\mathrm{P}<$ 0.05).

\section{Validation of candidate miRNAs}

The elevation of miR-134 and miR-410 was validated using TaqMan miRNA qRT-PCR in all participants
Table 1 Alterations in plasma microRNA levels in patients with an acute pulmonary embolism compared to healthy controls

\begin{tabular}{|c|c|c|c|}
\hline microRNA & Regulation & Fold Change & p-value \\
\hline hsa-miR-134 & up & 25.392 & 0.047 \\
\hline hsa-miR-410 & up & 15.221 & 0.028 \\
\hline hsa-miR-520g & up & 6.768 & 0.048 \\
\hline hsa-miR-485-3p & up & 6.170 & 0.018 \\
\hline hsa-miR-362-5p & up & 5.987 & 0.047 \\
\hline hsa-miR-382 & up & 5.501 & 0.042 \\
\hline hsa-miR-548b-5p & up & 5.473 & 0.039 \\
\hline hsa-miR-139-3p & up & 5.049 & 0.024 \\
\hline hsa-miR-197 & up & 4.363 & 0.015 \\
\hline hsa-miR-574-3p & up & 4.303 & 0.024 \\
\hline hsa-miR-190 & up & 4.031 & 0.039 \\
\hline hsa-miR-489 & up & 3.656 & 0.048 \\
\hline hsa-miR-146a & up & 3.593 & 0.030 \\
\hline hsa-miR-320 & up & 2.470 & 0.033 \\
\hline hsa-miR-133a & up & 2.202 & 0.042 \\
\hline hsa-miR-20a* & down & 5.924 & 0.005 \\
\hline hsa-miR-7 & down & 4.990 & 0.010 \\
\hline hsa-miR-340* & down & 4.810 & 0.039 \\
\hline hsa-miR-923 & down & 4.255 & 0.036 \\
\hline hsa-let-7b & down & 4.068 & 0.000 \\
\hline hsa-let-7g & down & 3.512 & 0.009 \\
\hline hsa-miR-768-3p & down & 3.166 & 0.036 \\
\hline hsa-miR-30e & down & 3.010 & 0.011 \\
\hline hsa-miR-610 & down & 2.852 & 0.045 \\
\hline hsa-miR-126* & down & 2.799 & 0.006 \\
\hline hsa-miR-301a & down & 2.740 & 0.007 \\
\hline hsa-miR-30d & down & 2.720 & 0.004 \\
\hline hsa-miR-30a & down & 2.212 & 0.003 \\
\hline hsa-miR-374a & down & 2.185 & 0.012 \\
\hline hsa-miR-632 & down & 1.706 & 0.043 \\
\hline
\end{tabular}

(Figure 1A-B). The basic clinical characteristics of APE patients and non-APE patients are shown in Table 2. There were no significant differences in gender composition and age between the APE and non-APE groups. The change in candidate miRNAs for the APE group or nonAPE group versus the healthy controls is shown in Figure 1. These data have been normalized by the expression levels of miR-16, a widely used endogenous reference miRNA that was also confirmed to be unchanged in our microarrays. In addition, compared to cel-miR-39, miR16 is stable (Additional file 1 Figure S1). Moreover, we compared the difference in miR-134 expression between 10 APE patients and 10 healthy controls and obtained the same differences in miR-134 expression regardless of whether cel-miR-39 or miR-16 were used as the normalizer (Figure 2), which further supports that miR-16 is a stable reference in this study. 


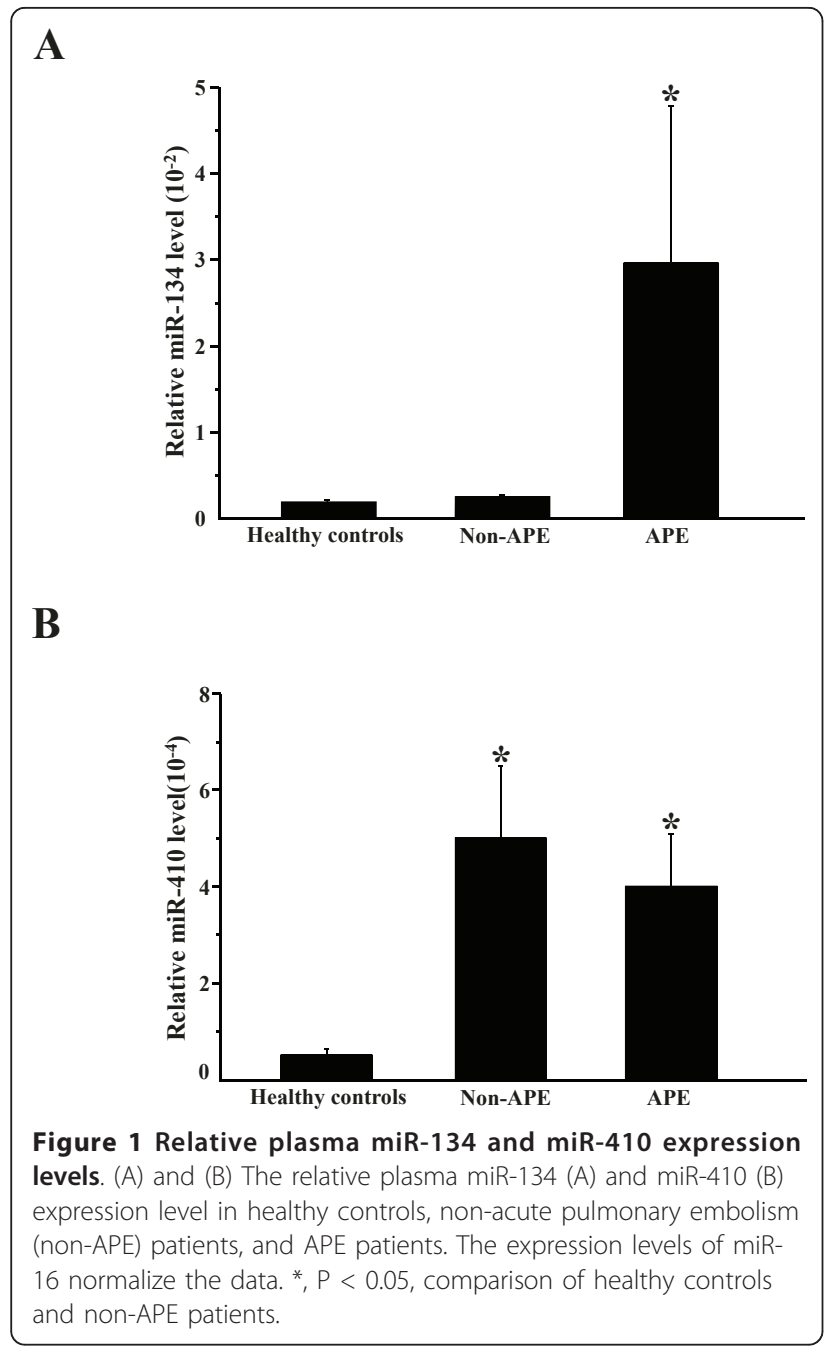

Table 2 Clinical characteristics of acute pulmonary embolism (APE) patients and non-APE patients

\begin{tabular}{|c|c|c|}
\hline Characteristics & APE $(n=32)$ & Non-APE $(n=22)$ \\
\hline Age (years) & $54.78 \pm 16.20$ & $62.27 \pm 23.33$ \\
\hline Male/female $(n / n)$ & $15 / 17$ & $10 / 12$ \\
\hline \multicolumn{3}{|l|}{ Risk stratification } \\
\hline High risk (n) & 1 & / \\
\hline Intermediate risk (n) & 15 & / \\
\hline Low risk $(n)$ & 16 & / \\
\hline \multicolumn{3}{|l|}{ Main clinical symptoms } \\
\hline Dyspnea & 10 & $22^{*}$ \\
\hline Thoracic pain & 11 & $18^{*}$ \\
\hline Clinical signs of DVT & 15 & $0^{*}$ \\
\hline \multicolumn{3}{|l|}{ Risk factors } \\
\hline Cancer & 4 & 2 \\
\hline Recent surgery ( $<30$ days) & 3 & 2 \\
\hline Previous DVT & 9 & $0^{*}$ \\
\hline Previous PE & 8 & $0^{*}$ \\
\hline
\end{tabular}

*, $\mathrm{P}<0.05$. DVT, Deep vein thrombosis; $\mathrm{PE}$, pulmonary embolism.

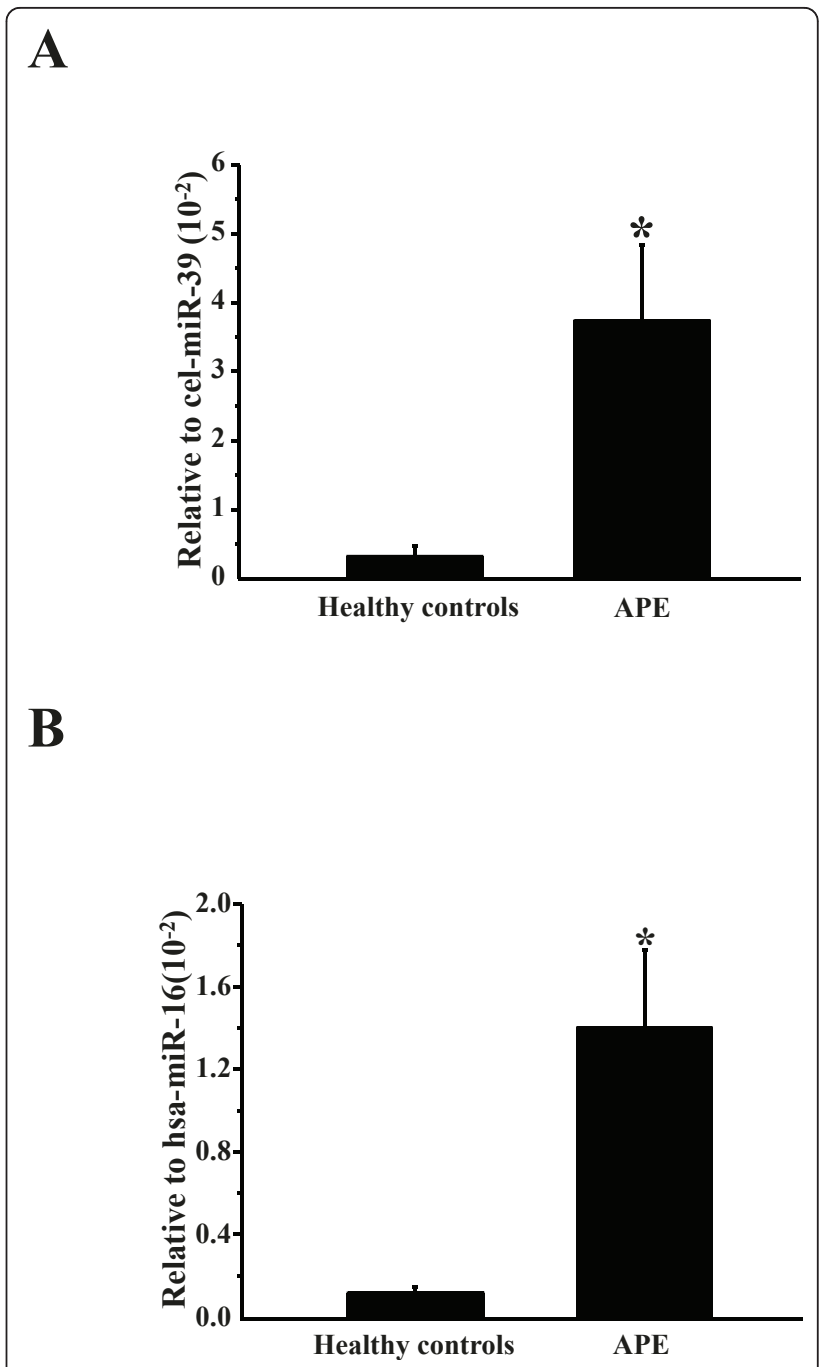

Figure 2 Relative plasma miR-134 expression levels normalized by cel- miR-39 and hsa-miR-16. (A) and (B) The relative plasma miR-134 expression levels normalized by cel-miR-39 (A) and hsamiR-16 (B) in healthy controls and acute pulmonary embolism patients. ${ }^{*}, P<0.05$, comparison with healthy controls.

The plasma miR-134 level was increased in the APE group compared to both healthy controls and the nonAPE group (Figure 1A). However, plasma miR-410 was also increased in the non-APE group (Figure 1B), indicating that miR-410, although initially identified by comparing the APE group to the healthy controls with miRNA microarrays, was also elevated in the non-APE group and therefore was not further studied. To confirm that the assay is reproducible, we also analyzed miR-134 expression level in the plasma (second sampling) collected $1 \mathrm{~h}$ after the first sampling of the plasma of three APE patients. No significant difference in the level of miR-134 was found between the first sampling and the second sampling, which indicates that the assay is reproducible (Additional file 2 Figure S2). We also compared 
the plasma miR-134 level between high-intermediaterisk APE and low-risk APE and found that the miR-134 level was significantly higher in the high-intermediate-risk APE patients compared to low-risk patients (Figure 3A).

\section{Diagnostic accuracy of plasma miR-134 for APE}

The ROC curve analysis was used to analyze the diagnostic accuracy of plasma miR-134. When a comparison was made between the APE group and the healthy controls, the AUC was 0.833 (95\% confidence interval, 0.737 to 0.929 ; $\mathrm{P}<0.001$ ) (Figure $4 \mathrm{~A}$ ). When a comparison was made between the APE group and the non-APE group, the AUC was 0.756 (95\% confidence interval, 0.629 to $0.883 ; \mathrm{P}=0.002$ ). Using 0.00272375 as a cutoff value for the plasma miR-134 relative expression level, the sensitivity and specificity of miR-134 for the diagnosis of APE in patients reporting dyspnea, chest pain, or cough were $68.8 \%$ and $68.2 \%$, respectively (Figure $4 \mathrm{~B}$ ). In addition, plasma miR-134 distinguished APE cases from healthy controls plus non-APE cases with an AUC of 0.802 (95\% confidence interval, 0.702 to 0.901 ; $\mathrm{P}<$ 0.001) (Figure 4C).

\section{Discussion}

APE has a nonspecific clinical presentation and is difficult to diagnose [24]. Although there has been great progress in the detection and exclusion of APE with the advent of D-dimer testing and chest CT scans, there is still a great need for simple and reliable biomarker detection for objective diagnostic testing of APE $[7,31]$.

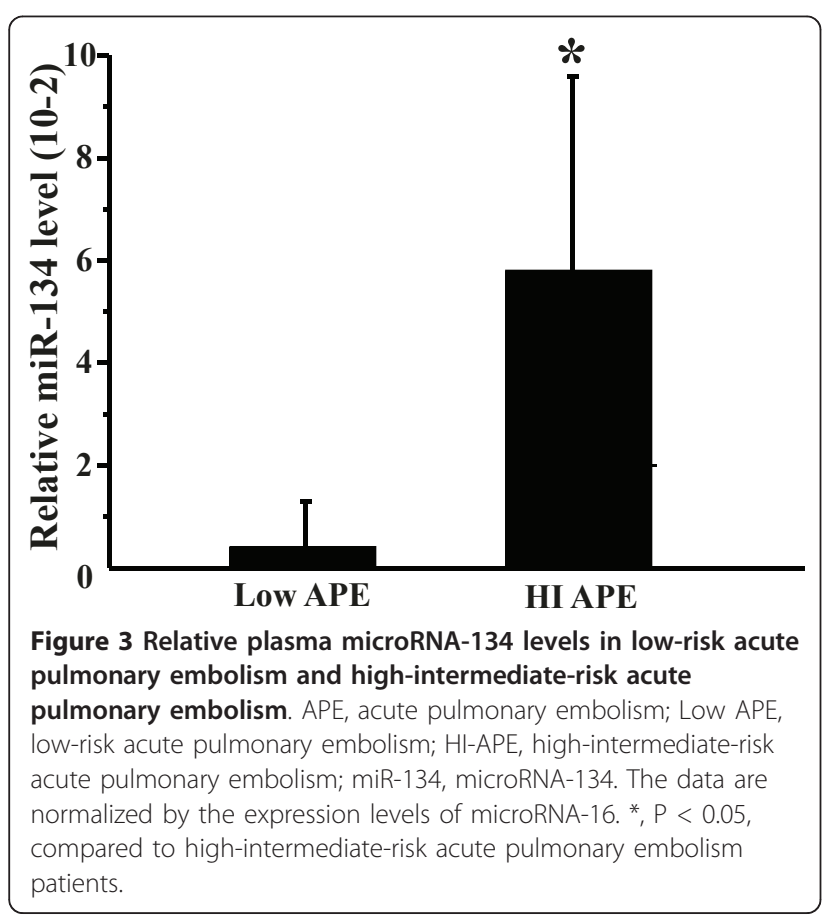

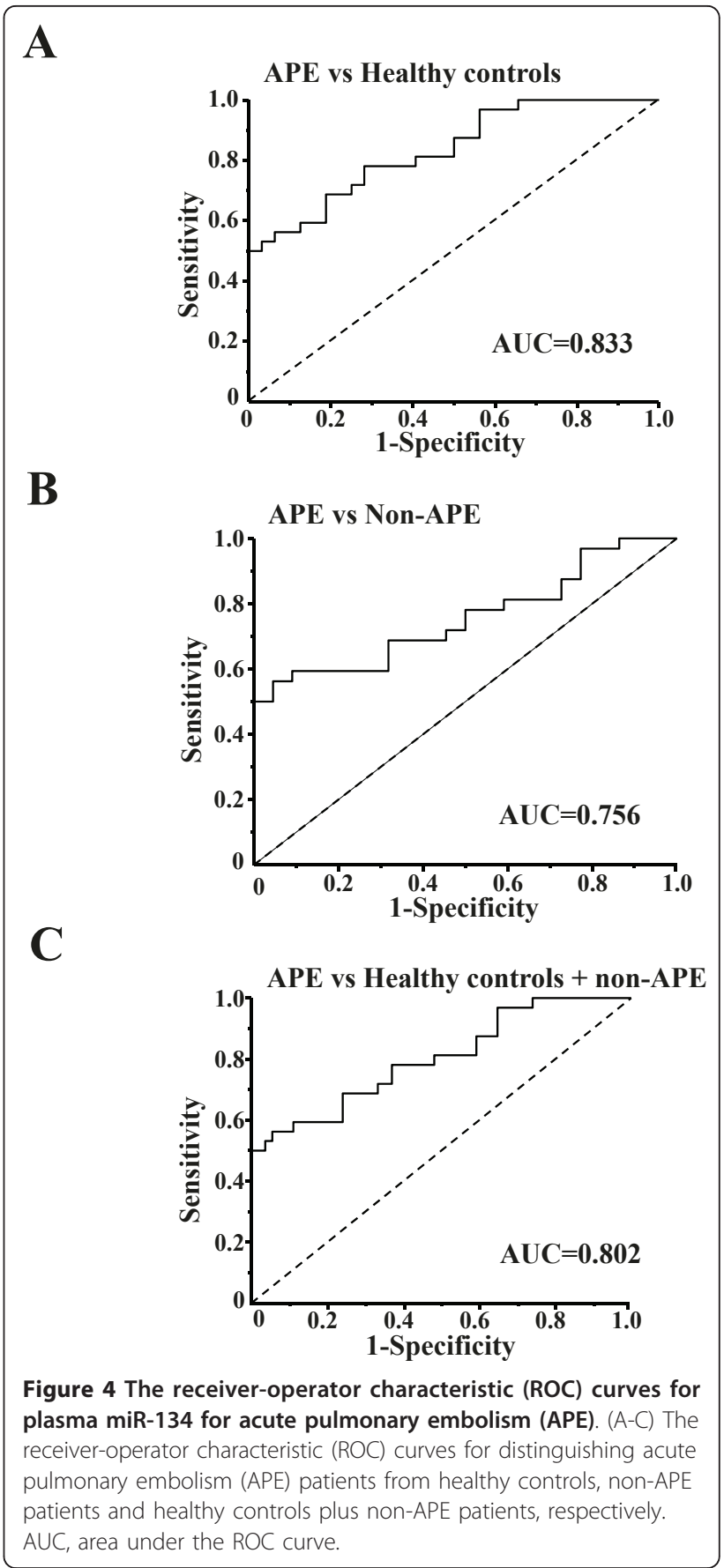

In this study, we have identified plasma miR-134 as a potential biomarker for APE.

Ideally, a biomarker should be reproducible and have a high sensitivity and specificity for the diagnosis of a specific disease [10]. miRNAs are exciting potential biomarkers because they fulfill many of these criteria $[10,16]$. Interestingly, miRNAs are present in human plasma in a remarkably stable form that is protected from endogenous RNase activity and remain stable even 
after being subjected to harsh conditions [15,32]. The stability, low structural complexity, and lack of post-processing modifications make plasma miRNAs ideal biomarker candidates [16]. The high sensitivity and specificity of miRNA detection using qRT-PCR may create precise cutoff concentrations for diagnosis [15,17]. To date, the role of plasma miRNAs in APE has not been reported. As demonstrated by the miR-134 identified in this study, the use of miRNAs as minimally invasive and robust biomarkers would result in incredible breakthroughs for the diagnosis of this common disease.

Spiral CT arteriography is widely used in the clinic. The sensitivity of spiral CT arteriography alone was $83 \%$, whereas the combination of CT arteriography and CT venography increased the sensitivity to $90 \%$. However, in those patients with unavailable multidetector $\mathrm{CT}$, renal failure or an allergy to contrast dye, plasma miR-134 might be an ideal alternative. In addition, using plasma miR-134 detection reduces the overall radiation exposure compared to CT $[1,4,24]$.

We have demonstrated that the plasma miR-134 level was not affected by non-APE conditions. Plasma miR134 could distinguish APE cases from healthy controls or non-APE cases with an AUC of 0.833 or 0.756 , respectively, which indicates the potential for using plasma miR-134 as a biomarker to diagnose APE. Interestingly, a recent report indicated that the expression of miR-134 was 3.5-fold higher in the peripheral blood mononuclear cells of unstable angina pectoris patients compared to those with stable angina pectoris [33]. In this study, the plasma miR-134 level in 7 patients with unstable angina pectoris and 3 with an acute myocardial infarction was similar to healthy controls. This discrepancy may have been due to the difference between miRNA levels in plasma versus peripheral mononuclear cells used in the original report [33]. In addition, Wong et al. found an increased expression of miR-134 in patients with squamous cell carcinoma. However, their study used tissue, whereas our study used plasma [26].

Our study was subject to a number of limitations. First, the number of APE patients in this study is relatively small. The results obtained in this small group will require replication in large, independent studies of APE. Second, it would be interesting to analyze the relationship of miR-134 to the laboratory findings in both APE and non-APE patients. For example, it would be helpful to investigate whether combining the values of $\mathrm{D}$-dimer and miR-134 would greatly enhance the specificity and sensitivity for plasma miR-134. Some non-APE patients in this study did not have D-dimer results because all of the collected plasma was used to isolate total RNA. Further studies are needed to resolve this issue. However, the D-dimer test has a high sensitivity but is non-specific because it is also positive in patients with infection, cancer, and other inflammatory diseases $[1,24]$. The specificity of miR-134 in diagnosing APE was better than D-dimer test in this study. Third, further work is required to determine the additive benefit of miR-134 in algorithms for APE detection that incorporate other diagnostic modalities in prospective fashion. Fourth, whether circulating miRNAs have a biological function is unclear. It is commonly speculated that circulating miRs play a role in maintaining the homeostasis of the circulatory system [30]. Whether plasma miR-134 can trigger some pathogenic or protective effects in APE remains uncertain. Finally, the pathobiological mechanism of miR-134 levels and the relationship with APE is unclear. Prior studies have demonstrated that the release of miRNA from exosomes, microparticles, or apoptotic bodies may be the cause of miRNA dysregulation in disease [24,34].

\section{Conclusions}

In conclusion, we have found that elevated plasma miR134 levels are a potential novel biomarker for the diagnosis of APE. Our results provide a basis for future efforts to develop plasma miRNA-based assays to diagnose APE.

\section{Additional material}

Additional file 1: Figure S1 - Relative plasma miR-16 levels in acute pulmonary embolism patients. APE, acute pulmonary embolism. Additional file 2: Figure S2 - Relative plasma microRNA-134 levels in the first sampling and second sampling. n.S., not significant.

\section{Acknowledgements}

This work was supported by the "973" Program Fund of China (2007CB512100 and 2011CB504006); the Yangtze Scholars Program Fund by the Ministry of Education, China; the Program Fund for Innovative Research Teams by the Ministry of Education, China; the Major Project Fund of Science and Techonology Commission of Shanghai Municipality (10JC1414700) (all to Y.-H.C.); the Youth Fund of the Key Laboratory of Arrhythmias, Ministry of Education, China (East Hospital, Tongji University School of Medicine) (to DDL and $\mathrm{HZ}$ ); and National Institutes of Health grants (R01HL104156, R21DA027021, R01HL092577, to P.T.E).

\section{Author details}

${ }^{1}$ Key Laboratory of Arrhythmias, Ministry of Education, China (East Hospital, Tongji University School of Medicine), Shanghai, China. ${ }^{2}$ Institute of Medical Genetics, Tongji University, Shanghai, China. ${ }^{3}$ Department of Pulmonary Circulation, Shanghai Pulmonary Hospital, Tongji University, Shanghai, China. ${ }^{4}$ Cardiovascular Research Center and Cardiac Arrhythmia Service,

Massachusetts General Hospital, Boston, USA. ${ }^{5}$ Department of Medicine, University of California San Diego, La Jolla, USA. ${ }^{6}$ Department of Cellular and Molecular Medicine, Carol Davila University of Medicine and Pharmacy, Bucharest, Romania.

\section{Authors' contributions}

JJX and DDL conducted the TaqMan microRNA array; ZCJ, XLC and LP enrolled patients and recorded clinical data; $H Z, Y L$, and $Y L$ performed the TaqMan-based miRNA quantitative real-time reverse transcription polymerase chain reactions; LYP, XQL, and YFS performed the statistical analysis and reviewed the manuscript; PTE, RL, LMP and GC analyzed the data and reviewed the manuscript. YHC designed the study and wrote the manuscript. All authors read and approved the final manuscript. 


\section{Competing interests}

The authors declare that they have no competing interests.

Received: 5 March 2011 Accepted: 24 September 2011

Published: 24 September 2011

\section{References}

1. Agnelli G, Becattini C: Acute pulmonary embolism. N Engl I Med 2010, 363:266-274

2. Horlander KT, Mannino DM, Leeper KV: Pulmonary embolism mortality in the United States, 1979-1998: an analysis using multiple-cause mortality data. Arch Intern Med 2003, 163:1711-1717.

3. Goldhaber SZ, Elliott CG: Acute pulmonary embolism: part I: epidemiology, pathophysiology, and diagnosis. Circulation 2003, 108:2726-2729.

4. Cohen AT, Agnelli G, Anderson FA, Arcelus Jl, Bergqvist D, Brecht JG, Greer IA, Heit JA, Hutchinson JL, Kakkar AK, Mottier D, Oger E, Samama MM, Spannagl M: Venous thromboembolism (VTE) in Europe. The number of VTE events and associated morbidity and mortality. Thromb Haemost 2007, 98:756-764.

5. Perrier A, Nendaz MR, Sarasin FP, Howarth N, Bounameaux H: Costeffectiveness analysis of diagnostic strategies for suspected pulmonary embolism including helical computed tomography. Am J Respir Crit Care Med 2003, 167:39-44

6. Schoepf UJ, Goldhaber SZ, Costello P: Spiral computed tomography for acute pulmonary embolism. Circulation 2004, 109:2160-2167.

7. Kearon C: Diagnosis of pulmonary embolism. CMAJ 2003, 168:183-194.

8. Zampetaki A, Kiechl S, Drozdov I, Willeit P, Mayr U, Prokopi M, Mayr A, Weger S, Oberhollenzer F, Bonora E, Shah A, Willeit J, Mayr M: Plasma microRNA profiling reveals loss of endothelial mir-126 and other microRNAs in type 2 diabetes. Circ Res 2010, 107:810-817.

9. Contu R, Latronico MVG, Condorelli G: Circulating microRNAs as potential biomarkers of coronary artery disease: a promise to be fulfilled? Circ Res 2010, 105:573-574.

10. Alevizos I, Illei GG: MicroRNAs as biomarkers in rheumatic diseases. Nature Rev Rheumatol 2010, 6:391-398.

11. van Rooij E, Olson EN: Searching for miR-acles in cardiac fibrosis. Cir Res 2009, 104:138-140

12. D'Alessandra Y, Devanna P, Limana F, Straino S, Di Carlo A, Brambilla PG Rubino M, Carena MC, Spazzafumo L, De Simone M, Micheli B, Biglioli P, Achilli F, Martelli F, Maggiolini S, Marenzi G, Pompilio G, Capogrossi MC: Circulating microRNAs are new and sensitive biomarkers of myocardial infarction. Eur Heart J 2010, 31:2765-2773.

13. Voellenkle C, van Rooij J, Cappuzzello C, Greco S, Arcelli D, Di Vito L, Melillo G, Rigolini R, Costa E, Crea F, Capogrossi MC, Napolitano M, Martelli F: MicroRNA signatures in peripheral blood mononuclear cells of chronic heart failure patients. Physiol Genomics 2010, 42:420-426.

14. Markham DW, Hill JA: MicroRNAs and heart failure diagnosis: MiR-acle or MiR-age? Circ Res 2010, 106:1011-1013.

15. Mitchell PS, Parkin RK, Kroh EM, Fritz BR, Wyman SK, PogosovaAgadjanyan EL, Peterson A, Noteboom J, O'Briant KC, Allen A, Lin DW, Urban N, Drescher CW, Knudsen BS, Stirewalt DL, Gentleman R, Vessella RL, Nelson PS, Martin DB, Tewari M: Circulating microRNAs as stable bloodbased markers for cancer detection. Proc Natl Acad Sci USA 2008, 105:10513-10518.

16. Wang K, Zhang S, Marzolf B, Troisch P, Brightman A, Hu Z, Hood LE, Galas DJ: Circulating microRNAs, potential biomarkers for drug-induced liver injury. Proc Natl Acad Sci USA 2009, 106:4402-4407.

17. Ai J, Zhang R, Li Y, Pu J, Lu Y, Jiao J, Li K, Yu B, Li Z, Wang R, Wang L, Li Q, Wang N, Shan H, Li Z, Yang B: Circulating microRNA-1 as a potential novel biomarker for acute myocardial infarction. Biochem Biophys Res Commun 2010, 391:73-77.

18. Fichtlscherer S, De Rosa S, Fox H, Schwietz T, Fischer A, Liebetrau C, Weber M, Hamm CW, Röxe T, Müller-Ardogan M, Bonauer A, Zeiher AM, Dimmeler S: Circulating microRNAs in patients with coronary artery disease. Circ Res 2010, 107:677-684.

19. Adachi T, Nakanishi M, Otsuka Y, Nishimura K, Hirokawa G, Goto Y, Nonogi $\mathrm{H}$, Iwai N: Plasma microRNA 499 as a biomarker of acute myocardial infarction. Clin Chem 2010, 56:1183-1185.
20. Wang GK, Zhu JQ, Zhang JT, Li Q, Li Y, He J, Qin YW, Jing Q: Circulating microRNA: a novel potential biomarker for early diagnosis of acute myocardial infarction in humans. Eur Heart J 2010, 31:659-666.

21. Tijsen AJ, Creemers EE, Moerland PD, de Windt $L$, van der Wal AC, Kok WE, Pinto YM: MiR423-5p as a circulating biomarker for heart failure. Circ Res 2010, 106:1035-1039.

22. Mishra PK, Tyagi N, Kumar M, Tyagi SC: MicroRNAs as a therapeutic target for cardiovascular diseases. J Cell Mol Med 2009, 13:778-789.

23. Liu J, van Mil A, Vrijsen K, Zhao J, Gao L, Goumans MJ, Doevendans PA, Sluijter JP: MicroRNA-155 prevents necrotic cell death in human cardiomyocyte progenitor cells via targeting RIP1. J Cell Mol Med 2010.

24. Torbicki A, Perrier A, Konstantinides S, Agnelli G, Galiè N, Pruszczyk P, Bengel F, Brady AJ, Ferreira D, Janssens U, Klepetko W, Mayer E, RemyJardin M, Bassand JP, Vahanian A, Camm J, De Caterina R, Dean V, Dickstein K, Filippatos G, Funck-Brentano C, Hellemans I, Kristensen SD, McGregor K, Sechtem U, Silber S, Tendera M, Widimsky P, Zamorano JL, Zamorano $J$, et al: Guidelines on the diagnosis and management of acute pulmonary embolism: the Task Force for the Diagnosis and Management of Acute Pulmonary Embolism of the European Society of Cardiology (ESC). Eur Heart J 2008, 29:2276-2315.

25. Lawrie CH, Gal S, Dunlop HM, Pushkaran B, Liggins AP, Pulford K, Banham AH, Pezzella F, Boultwood J, Wainscoat JS, Hatton CS, Harris AL: Detection of elevated levels of tumour-associated microRNAs in serum of patients with diffuse large B-cell lymphoma. Br J Haematol 2008, 141:672-675.

26. Wong TS, Liu XB, Wong BY, Ng RW, Yuen AP, Wei WI: Mature miR-184 as Potential Oncogenic microRNA of Squamous Cell Carcinoma of Tongue. Clin Cancer Res 2008, 14:2588-2592.

27. Huang Z, Huang D, Ni S, Peng Z, Sheng W, Du X: Plasma microRNAs are promising novel biomarkers for early detection of colorectal cancer. Int J Cancer 2010, 127:118-126.

28. Liu CJ, Kao SY, Tu HF, Tsai MM, Chang KW, Lin SC: Increase of microRNA miR-31 level in plasma could be a potential marker of oral cancer. Oral Dis 2010, 16:360-364

29. Yamamoto Y, Kosaka N, Tanaka M, Koizumi F, Kanai Y, Mizutani T, Murakami Y, Kuroda M, Miyajima A, Kato T, Ochiya T: MicroRNA-500 as a potential diagnostic marker for hepatocellular carcinoma. Biomarkers 2009, 14:529-538.

30. Reid G, Kirschner MB, van Zandwijk N: Circulating microRNAs: Association with disease and potential use as biomarkers. Crit Rev Oncol Hematol 2010.

31. Stein PD, Fowler SE, Goodman LR, Gottschalk A, Hales CA, Hull RD, Leeper KV Jr, Popovich J Jr, Quinn DA, Sos TA, Sostman HD, Tapson VF, Wakefield TW, Weg JG, Woodard PK: Multidetector computed tomography for acute pulmonary embolism. N Engl J Med 2006, 354:2317-2327.

32. Dimmeler S, Zeiher AM: Circulating microRNAs: novel biomarkers for cardiovascular diseases? Eur Heart J 2010, 31:2705-2707.

33. Hoekstra M, van der Lans CA, Halvorsen B, Gullestad L, Kuiper J, Aukrust P, van Berkel TJ, Biessen EA: The peripheral blood mononuclear cell microRNA signature of coronary artery disease. Biochem Biophys Res Commun 2010, 394:792-797.

34. Skog J, Würdinger T, van Rijn S, Meijer DH, Gainche L, Sena-Esteves M, Curry WT Jr, Carter BS, Krichevsky AM, Breakefield XO: Glioblastoma microvesicles transport RNA and proteins that promote tumour growth and provide diagnostic biomarkers. Nat Cell Biol 2008, 10:1470-1476.

doi:10.1186/1479-5876-9-159

Cite this article as: Xiao et al:: MicroRNA-134 as a potential plasma biomarker for the diagnosis of acute pulmonary embolism. Journal of Translational Medicine 2011 9:159. 